Embedding Physical Activity into the Healthcare Curriculum - A Case Study

Karen Milton $^{\mathrm{a} *}$, Jonathan Larner ${ }^{\mathrm{b}}$, Sarah Hanson ${ }^{\mathrm{b}}$, Andy Jones ${ }^{\mathrm{a}}$

${ }^{a}$ Norwich Medical School, Faculty of Medicine and Health Sciences, University of East

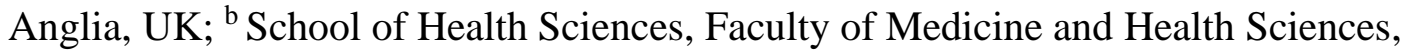
University of East Anglia, UK

* corresponding author

Dr Karen Milton

Norwich Medical School

University of East Anglia

Norwich

NR4 7TJ

k.milton@uea.ac.uk

Keywords: Physical activity, teaching, curriculum, medicine, health Word count: 1878 


\section{Abstract (max 200 words)}

Physical inactivity is a key risk factor for a wide range of non-communicable diseases, yet a large proportion of the population fail to meet recommended physical activity levels. Healthcare has been identified as a key setting in which to intervene to encourage physical activity behaviour change. However, those working in the health care sector must be provided with training opportunities to increase knowledge, competence and motivation. We reviewed the structure and content of the current health-related courses at the University of East Anglia to identify opportunities to deliver more content on physical activity. We identified five areas for action: the development of new learning outcomes; the creation and delivery of taught sessions on physical activity; providing resources to faculty members to support the integration of the topic across all relevant modules and courses; the development of electronic resources for students; and modifications to the Problem-Based Learning (PBL) scenarios. Taking a multi-faceted and integrated approach was important to avoid physical activity being viewed as a 'bolt-on' topic. It also helped to maximise exposure to the topic while minimising disruption to the course structure and timetable. The actions taken have proved feasible and have been well received by students and staff. 


\section{Background}

Physical inactivity is a key risk factor for a wide range of non-communicable diseases (NCDs) including cardiovascular disease, stroke, diabetes, and cancer. ${ }^{1}$ In addition, being regularly physically active is associated with improved mental health, sleep and cognitive function. ${ }^{1}$ To achieve these benefits adults are recommended to undertake a minimum of 150 minutes per week of at least moderate-intensity aerobic activity and to undertake activities to strengthen muscles twice per week. ${ }^{2,3}$ Data indicate, however, that just $31 \%$ of men and $23 \%$ of women in England met the physical activity guidelines in $2016 .{ }^{4}$ As such, evidence-based interventions are needed to tackle population levels of inactivity.

Healthcare has been identified as a key setting in which to intervene to encourage physical activity behaviour change. In developed countries, $70-80 \%$ of adults visit their general practitioner (GP) at least once per year, and patients with chronic disease such as diabetes, or risk factors for cardiovascular disease such as hypertension, are reviewed regularly. ${ }^{5}$ In addition to the extensive population reach of healthcare professionals, they are widely respected and trusted, meaning they have considerable potential to influence public and individual opinion. ${ }^{6,7}$ Indeed, one in four people say they would be more active if advised by a GP or nurse. ${ }^{8}$

Research has shown that a large proportion of final year medical students are unaware of the physical activity recommendations and $80 \%$ stated that they had not received training in lifestyle medicine. ${ }^{9}$ Among practising doctors, as many as $72 \%$ do not discuss the benefits of physical activity with their patients. ${ }^{10}$ Given that qualified doctors, nurses and other allied health professionals may see half a million patients during their career, this presents a hugely underutilised opportunity for health promotion. ${ }^{11}$ Doctors, in particular, have cited a lack of 
training, knowledge, confidence, and time as barriers to discussing physical activity with their patients. ${ }^{12}$ Furthermore, it has been reported that health professionals in primary care consider physical activity to be beyond their remit and less important than other health promotion activities such as smoking cessation. ${ }^{14}$

The General Medical Council has identified the promotion of lifestyle changes as a key outcome for graduates. ${ }^{15}$ This is supported by the Global Action Plan on Physical Activity, which states the need to strengthen pre- and in-service training of health professionals to increase knowledge and skills. ${ }^{16}$ In turn, health professionals should be competent to undertake assessment and counselling on physical activity in routine practice and to advise patients on appropriate opportunities to increase their activity levels.

The Faculty of Medicine and Health Sciences at the University of East Anglia (UEA) prides itself on innovative teaching and research in health, and many of the health related courses at the UEA have been rated among the top 10 in the United Kingdom (UK). ${ }^{17}$ At the UEA a range of actions have been implemented to address the knowledge gaps around physical activity among students of health-related courses. This paper highlights the actions that have been undertaken at the UEA over the past 18 months, with the aim of sharing practice and encouraging other universities to place greater emphasis on physical activity within the curricular of health-related courses.

\section{Methods}

A group of academics from the Faculty of Medicine and Health Sciences (comprising the four authors) agreed to form a leadership group for this agenda. We reviewed the structure and the content of the current pre-registration health-related courses to identify opportunities 
to deliver more content on physical activity. We collectively identified five areas for action that we felt could be tackled immediately: the development of new learning outcomes; the creation and delivery of taught sessions on physical activity; providing resources to faculty members to support the integration of the topic across all relevant modules and courses; the development of electronic resources for students; and modifications to the Problem-Based Learning (PBL) scenarios. Details of the actions undertaken in each area are provided below.

\section{The Development of New Learning Outcomes}

As with any curriculum, the content of the health-related educational programmes at the UEA is based on a comprehensive set of learning outcomes. As such, ensuring the topic of physical activity features within the learning outcomes of relevant courses was critical to ensuring that it gets taught on the curriculum. We have developed a range of learning outcomes specific to physical activity including:

- Appreciate the importance of physical inactivity as a risk factor for non-communicable diseases

- Explain the recommended volume of physical activity for health benefits

- Describe the health benefits of physical activity for specific conditions

- Describe the importance of the healthcare setting for physical activity promotion

- Explain the practical steps involved in promoting physical activity in healthcare

\section{The Creation and Delivery of Taught Sessions on Physical Activity}

At the UEA we have created new taught sessions on the topic of physical activity and health. For example, first-year medical students now receive two lectures, one on the links between physical activity and health, and one on the role of the medical profession in assessing and counselling on physical activity. The latter introduces students to the Moving Medicine on- 
line platform, which provides information for health professionals on how to approach a discussion about physical activity with patients and how to undertake assessment and counselling on physical activity within the healthcare setting (movingmedicine.ac.uk). Our health courses now include the assessment of physical activity when taking histories from patients, as well as how to graduate physical activity as a therapeutic intervention for patients with varying capabilities.

\section{Providing Resources to Faculty Members}

In 2017, Public Health England (PHE) commissioned the development of a series of slide sets on the links between physical activity and a wide range of health conditions including heart disease and stroke, hypertension, diabetes, cancer, osteoporosis, sarcopenia, mental health, obesity and falls. The slide sets are managed by 'Exercise Works' and have been made available free of charge to universities across the UK. At the UEA, these slide sets have been shared via the online learning portal with teaching staff across the medical degree, who have been encouraged to embed physical activity into the teaching of all modules.

\section{The Development of Electronic Resources for Students}

For students, we have established a new area on our E-learning platform dedicated to physical activity and health. Content includes links to the UK and the Global physical activity guidelines, the Chief Medical Officer's (CMO) physical activity infographics, the Exercise Works slide sets, the Global Action Plan on Physical Activity, and links to the Moving Medicine website. Students on some health-related courses are sent a monthly online blog on physical activity, each of which promotes one of the Exercise Works resources and provides a summary of key information. 


\section{Modifications to Problem-Based Learning (PBL) Scenarios}

Much of the teaching at the UEA, and particularly on the medical programme, is done through Problem-Based Learning (PBL). This involves students being presented with cases and working together to determine the best course of action for each patient. We are reviewing the scenarios used in PBL to ensure that, in at least some scenarios, reference is made to the patient's physical activity level. This will help to establish physical activity as a 'vital sign' that should be considered when assessing the health status and needs of all patients. Physical activity behaviour change is also specifically being addressed in the Enquiry-Based Learning that is undertaken by physiotherapists and occupational therapists.

\section{Discussion}

The promotion of physical activity through healthcare is identified as a 'good buy' by the WHO, which recommends screening and counselling in routine practice. ${ }^{18}$ In order to deliver this, those working in the health care sector must be provided with training opportunities to increase knowledge and competence. Incorporating physical activity into the undergraduate curricular of health-related programmes presents an opportunity to deliver this training. Whilst the utility of physical activity promotion in the healthcare setting is clear, few examples have been shared on how to embed physical activity into the undergraduate curricular of relevant courses.

The Exercise Works slide sets provide a comprehensive overview of the links between physical activity and a wide range of health conditions. The amount of work required to produce such slide sets would be beyond the capacity of most faculty members, who might also lack the relevant background knowledge. Having these resources readily available provides faculty members with a clear and succinct overview of the evidence base. 
Furthermore, the comprehensiveness of the material, which in many cases may be too much to deliver in its entirety, means that faculty members are able to select the slides that they feel are most relevant and these can be 'slotted in' to existing teaching materials.

In addition to embedding the topic into each module, we have developed specific taught sessions on physical activity. These sessions provide an opportunity to discuss the practicalities of assessing and counselling on physical activity in routine care. Moving Medicine is a new resource developed to support health professionals in discussing physical activity with their patients. The web-platform allows the health professional to select either a specific condition or 'primary prevention' and to indicate how much time they have available within the consultation. The health professional is then directed to a step-by-step guide on how to raise the topic and the sorts of things to discuss with the patient, given their health status and the time available. This resource has been particularly well received by students.

At the UEA, a multi-faceted approach was taken to embedding physical activity into the curricular of the health and medical programmes. This was felt to be important to avoid physical activity being viewed as a 'bolt-on' topic. The benefits of physical activity for both prevention and treatment spans a broad range of health conditions, and as such, the topic has relevance across all modules of the medical degree. The topic also has relevance across much of the nursing, physiotherapy and occupational therapy programmes. The changes that have been introduced were based on a review of current practice at the UEA and were designed to maximise exposure to the topic while minimising disruption to the course structure and timetable. 
Our experience has been that it is very much possible to integrate physical activity into the curricular of health and medical programmes, and students have been positive about the inclusion of physical activity in the various curricular. Research is needed to better understand what works in terms of increasing health professionals' knowledge of physical activity and providing them with the skills and confidence to implement assessment and counselling on physical activity in routine practice. In order to build the evidence base it is essential that future efforts to embed physical activity into health-related courses are supported by robust process and outcome evaluation.

\section{Conclusions}

It is critical that health services are reoriented to place greater emphasis on prevention. As one of the key behavioural risk factors for NCDs, it is important that future health professionals receive training on how to assess and counsel patients on physical activity. At the UEA we have undertaken a range of actions to embed physical activity into the curricular of relevant health-related courses. Overall the actions have proved feasible and appear to have been well received by students and staff. Furthermore, the availability of ready-made and online resources (for example movingmedicine.ac.uk) will support the adoption of similar action in other universities. 


\section{Acknowledgements}

Not applicable

\section{Declaration of Interests}

The authors declare that they have no competing interests.

\section{Authors' contributions}

All authors contributed the the activities reported in this manuscript. KM drafted the manuscript. JL, SH, and AJ contributing to revising the text. All authors approved the final version. 


\section{References}

1. 2018 Physical Activity Guidelines Advisory Committee. 2018 Physical Activity Guidelines Advisory Committee Scientific Report. Washington, DC, USA; 2018.

2. World Health Organization. Global recommendations on physical activity for health. Geneva; 2010.

3. Department of Health. Start active, stay active. Strategy. London; 2011.

4. $\quad$ NHS Digital. Health Survey for England, 2016. Leeds, UK; 2017.

5. van Doorslaer E, Masseria C, Koolman X. Inequalities in access to medical care by income in developed countries. Can Med Assoc J. 2006;174(2):177-83.

6. Weiler R, Chew S, Coombs N. Physical activity education in the undergraduate curricula of all UK medical schools. Are tomorrow's doctors equipped to follow clinical guidelines? Br J Sports Med. 2012;46(14):1024-6.

7. Bull F, Schipper E, Jamrozik K, Blanksby B. How can and do Australian doctors promote physical activity? Prev Med. 1997;26(6):866-73.

8. Orrow G, Kinmouth AL, Sansrson S, Sutton A. Effectiveness of physical activity promotion based in primary care: a systematic review and meta-analysis of randomised controlled trials. BMJ. 2012;344;e1389.

9. Radenkovic D, Aswani R, Ahmad I, Kreindler J, Robinson R. Lifestyle medicine and physical activity knowledge of final year UK medical students. BMJ Open Sport and Exercise Medicine. 2019; 5:DOI:10.1136/bmjsem-2019-000518.

10. Chatterjee R, Chapman T, Brannan MGT, Varney J. GP's knowledge, use , and confidence in national physical activity and health guidelines and tools: a questionnaire-based survey of general practice inEngland. British Journal of General Practice. 2017;67(663):e668-e675.

11. Gates AB, Making every contact count for physical activity - for tomorrow's patients: 
the launch of the interdisciplinary undergraduate resources on exercise medicine and health in the UK. Br J Sports Med, 2016; 50(6):322-323.

12. Shuval K, Leonard T, Drope J, Katz DL, Patel AVm Maitin-Shephard M, Amir O, Grinstein A. Physical activity counselling in primary care: insights from public health and behavioural economics. CA: A Cancer Journal for Clinicians, 2017.

13 Rose SA, Poynter PS, Anderson JW, Noar SM, Conigliaro J. Physician weight loss advice and patient weight loss behaviour change: a literature review and meta-analysis of survey data. Int J Obes. 2013; 37:118-128.

14. Din N, Moore G, Murphy S, Wilkinson C, Williams N. Health professionals' perspectives on exercise referral and physical activity promotion in primary care: Findings from a process evaluation of the National Exercise Referral Scheme in Wales. Health Educ J. 2015;74(6):743-57.

15. General Medical Council (2018) Outcomes for graduates 2018. London: General Medical Council.

16. World Health Organization. Global Action Plan on Physical Activity 2018 - 2030 More active people for a healthier world. Geneva, Switzerland; 2018.

17. Complete University Guide. The complete university guide, 2017. London, UK; 2017.

18. World Health Organization. "Best Buys" and other recommended interventions for the prevention and control of noncommunicable diseases. Geneva, Switzerland; 2017. 